\title{
OLINK
}

$B|O S C| E N C E$

\section{Visualization and quantification of protein-protein interactions in cells and tissues}

Olink Bioscience has developed a protein detection assay that allows precise detection and quantification of proteins, protein interactions and modifications in fixed cells and tissue samples. Duolink immunoassay reagents are based on in situ proximity ligation assays $\left(\mathrm{PLA}^{\mathrm{T}}{ }^{\mathrm{TM}}\right)$, and the assay reports proteins or protein complexes as countable distinct fluorescent or chromogenic spots visualized by standard microscopy. This allows for robust validation of protein-interaction data to show an interaction in the correct cellular context at physiologically relevant expression levels.

To allow detailed studies of protein-protein interactions, we have developed Duolink ${ }^{\circledR}$, a product based on in situ PLA ${ }^{\mathrm{TM}}$. This makes it possible to visualize and quantify specific protein interaction events in situ with unprecedented specificity. Duolink detects proximal proteins in their normal context at normal expression levels and also allows rare events to be detected by generating cell-to-cell statistics. Here we describe how Duolink can be used to detect and quantify protein-protein interactions in cells and tissue, enabling highresolution deciphering of signal transduction events of both heteroand homodimeric complexes.

The Duolink reagents and in situ PLA technology

The Duolink user first applies primary target-specific antibodies to fixed cells or tissue sections. The cells can be cultured and fixed on microscopy slides and microtiter plates or prepared as cytospin slides. Two primary antibodies selected from two different host speciessuch as one mouse and one rabbit antibody - are used together with the generic Duolink species-specific secondary antibodies. These secondary antibodies contain unique DNA strands that template the hybridization of added oligonucleotides. When in close proximity $(<40$ $\mathrm{nm}$ ), the oligonucleotides are ligated by a ligase to form a circular template. This template, still anchored to the antibody, is subsequently amplified and detected using complementary labeled oligonucleotide probes $^{1,2}$. Detection is performed either with a fluorescent label, for fluorescence microscopy, or horseradish peroxidase (HRP), for brightfield detection. The resulting distinct spots are derived from singlemolecule protein interaction events, which are visualized using a standard microscope (Fig. 1).

Mats Gullberg \& Ann-Catrin Andersson

Olink Bioscience, Uppsala, Sweden

Correspondence should be addressed to M.G. (Mats.Gullberg@olink.com).

\section{Applying Duolink for protein interaction studies}

In an interaction experiment using Duolink, two primary antibodies are used, one targeting each of the investigated proteins in an expected interaction. This concept is illustrated in Figure 2, where an interaction between SMAD2/3 and SMAD4 proteins is visualized. It is of importance that the two antibodies bind under identical conditions

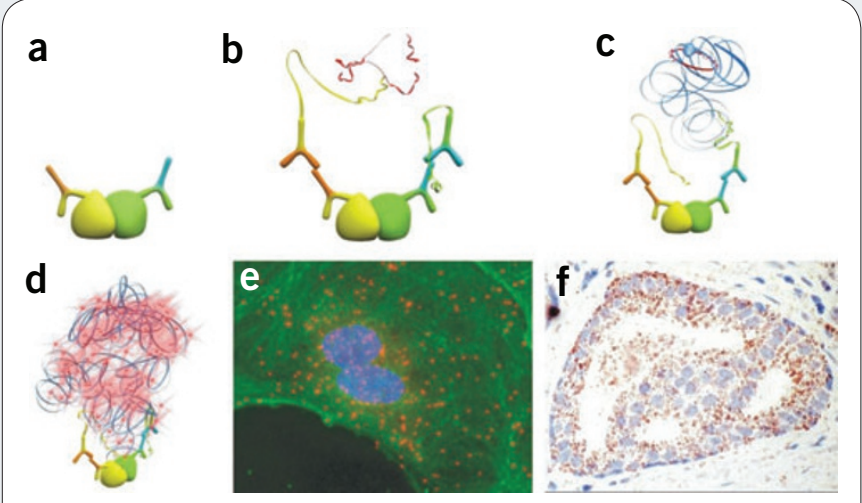

Figure 1 |The Duolink assay principle. (a) Two primary antibodies raised in different species and supplied by the user-for example, yellow/red (left) against the target protein and green/blue (right) against a phosphoepitope of the target-recognize the target antigen of interest. (b) Each species-specific secondary antibody provided in the Duolink kit has a different short DNA strand attached to it (yellow and green, respectively). When the secondary antibodies are in close proximity, the DNA strands can interact through a subsequent addition of two other circle-forming DNA oligonucleotides (red) (c) After joining of the two added oligonucleotides by enzymatic ligation, they are amplified via rolling-circle amplification using a polymerase. (d) After the amplification reaction, several hundred replications of the DNA circle have occurred, and labeled probes (added as Detection solution) highlight the product. (e,f) The resulting high concentration of fluorescence or HRPconverted chromogenic substrate in each single-molecule amplification product is easily visible as a distinct spot in a fluorescence microscope (e) or a bright-field microscope (f). Nuclei are stained with Hoechst (e) or hematoxylin (f) (blue) 

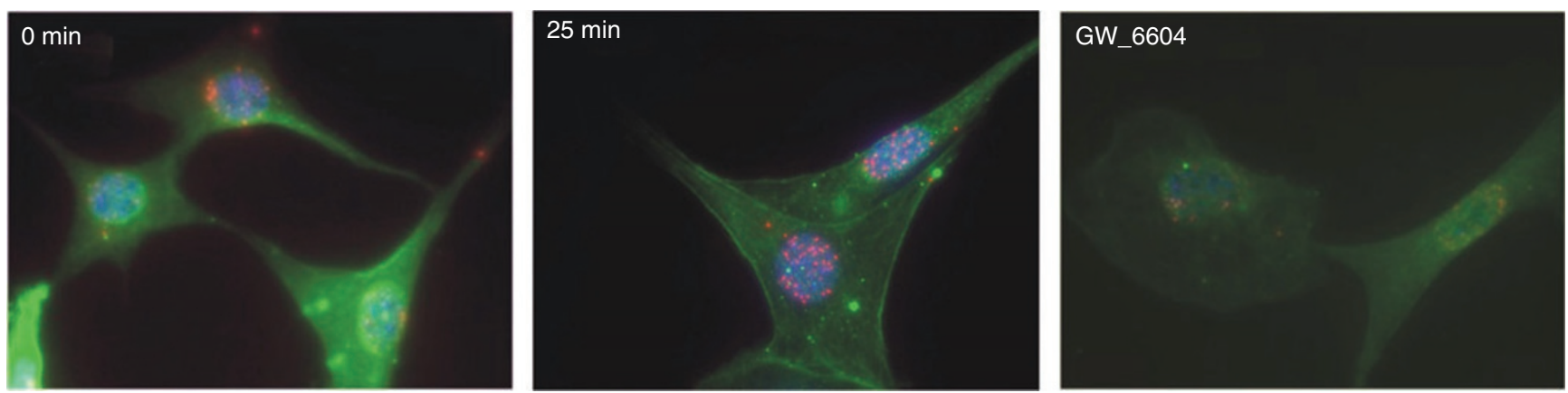

Figure 2 |Visualization of protein interactions. Images of mouse embryonic fibroblasts before (0 min) or after 25 min of TGF- $\beta$ stimulation ( 25 min), or treated with the known TGF- $\beta$ pathway inhibitor GW6604. One antibody was targeted against SMAD2/3 and one against SMAD4. Each PLA signal (red) is indicative of one detected interaction event. A clear increase in number of detected events is seen in the image showing cells 25 min after TGF- $\beta$ stimulation. Nuclei (blue) are stained with Hoechst 33342 and actin is stained with FITC anti-actin (green). Images are kindly provided by A. Zieba, Uppsala University.

and that they are also able to bind their target when the target protein is interacting with its partner.

A nice example of the use of Duolink to verify an expected interaction was recently published for the proteins COUP-TFII and Proxl ${ }^{3}$. The interaction was first demonstrated using coimmunoprecipitation (colP) on transfected HUVEC cells overexpressing COUP-TFII and Proxl, and a follow-up Duolink experiment showed that the interaction occurs in vivo. Importantly, using Duolink, the authors were able to show that the interaction also occurrs in HLDEC cells expressing the proteins at their physiological expression levels and that the interaction takes place at the expected cellular compartment, to further validate the data. Duolink would thus allow the interaction to be validated in situ without the need for generating transfected cell-lines. The specificity of the Duolink assay in the HLDECs was validated with siRNA knockdown using a specific or scrambled siRNA sequence.

\section{Studying transient receptor dimerizations}

Another recent report offers a good example of how Duolink can be used together with colP to provide new biological information ${ }^{4}$. Although colP detected VEGF-C ligand-induced heterodimerization, it failed to detectVEGF-A ligand-induced heterodimerization of receptors VEGFR2 and VEGFR3. The latter was, however, clearly detected using Duolink. Furthermore, the authors were able to utilize Duolink's unique feature of detecting homodimerization events. They thereby showed that VEGF-A induced homodimerization of only VEGFR2, whereas VEGF-C induced homodimerization of only VEGFR3, and both ligands were capable of inducing heterodimerization. By subjecting their images to quantitative image analysis, they also highlighted Duolink's ability to yield objectively quantifiable results. This allowed the authors to unambiguously characterize the efficiency of different VEGF isoforms in inducing receptor heterodimerization. This highlights the opportunities given by Duolink to study protein interactions at relevant expression levels and to complement colP data, yielding new biological information.

\section{Conclusions}

In situ PLA, using Duolink reagents, is a straightforward process for reporting protein interactions with very high specificity in their natural context at physiological expression levels, and also for reporting homodimerization events. This is an efficient and simple method for both validation and characterization of protein interactions. In addition, covalently modified-for example, ubiqutinylated and SUMOylated ${ }^{5}$ - proteins can also be studied in situ owing to the dual recognition format provided by Duolink.

The Duolink reagent series provides powerful yet simple means to quantitatively visualize post-translational modifications of proteins in fixed cells and tissue with exceptional specificity and sensitivity. There is no need to overexpress target proteins, and data interpretation is facilitated by countable spots, each representing a single-molecule event. The Duolink reagents are available with multiple species of primary antibodies to fit the unique application of the user.

1. Söderberg, O. et al. Direct observation of individual endogenous protein complexes in situ by proximity ligation. Nat. Methods 3, 995-1000 (2006).

2. Jarvius, M. et al. In situ detection of phoshporylated platelet-derived growth factor receptor using a generalized proximity ligation method. Mol. Cell. Proteomics 6, 1500-1509 (2007)

3. Yamazaki, T., Yoshimatsu, Y., Morishita, Y., Miyazono, K. \& Watabe, T. COUP-TFII regulates the functions of Prox 1 in lymphatic endothelial cells through direct interaction. Genes Cells 14, 425-434 (2009).

4. Nilsson, l. et al. VEGF receptor $2 / 3$ heterodimers detected in situ by proximity ligation on angiogenic sprouts. EMBO J. published online (11 March 2010).

5. Sehat, B. et al. SUMOylation mediates the nuclear translocation and signaling of the IGF-1 receptor. Sci. Signal. 3, ra10 (2010).

This article was submitted to Nature Methods by a commercial organization and has not been peer reviewed. Nature Methods takes no responsibility for the accuracy or otherwise of the information provided. 